\title{
HONGOS MICORRÍCICO-ARBUSCULARES ASOCIADOS A CHENOPODIACEAE EN DOS AMBIENTES SALINOS DE CÓRDOBA
}

\author{
ALEJANDRA BECERRA ${ }^{1}$, NORBERTO BARTOLONI², NOELIA COFRÉ ${ }^{1}$, FLORENCIA \\ SOTERAS ${ }^{1}$ y MARTA CABELLO 3
}

\begin{abstract}
Summary: Arbuscular mycorrhizal fungi associated with Chenopodiaceae from two saline environments of Cordoba province. Arbuscular mycorrhizal fungi (AMF) colonize approximately $82 \%$ of land plants in every ecosystem, even in extreme conditions such as saline soils. In the present work we report the arbuscular mycorrhizal colonization and its vertical root concentration in four Chenopodiaceae plants species (Allenrolfea patagonica, Atriplex argentina, Heterostachys ritteriana and Suaeda divaricata) at five different soil depths in two salines of central Argentina. All the plant species showed mycorrhizal colonization and varied significantly among plant species and sites for all soil depths. Atriplex argentina presented the highest mycorrhizal colonization at almost all soil depths $(0-50 \mathrm{~cm})$. In all plant species root concentration decrease as depth increases at both sites. The presence of mycorrhizal roots in the deeper layers of soil in the four Chenopodiaceae plants species, shows that these fungi are tolerant to live in the saline soils of central Argentina.
\end{abstract}

Key words: Arbuscular mycorrhizal fungi; saline environments; soil profile; vertical distribution.

Resumen: Los hongos micorrícico-arbusculares (HMA) colonizan aproximadamente el $82 \%$ de las plantas terrestres en cualquier ecosistema, aún en condiciones extremas tales como los suelos salinos. En el presente trabajo se reporta la colonización micorrícica arbuscular (CMA) y su concentración radical en cuatro especies de Chenopodiaceae (Allenrolfea patagonica, Atriplex argentina, Heterostachys ritteriana y Suaeda divaricata) en cinco profundidades del suelo en dos salinas del Centro de Argentina. Todas las plantas mostraron colonización micorrícica que varió significativamente entre especies y sitios para todas las profundidades. Atriplex argentina presentó el mayor porcentaje de CMA en casi todas las profundidades $(0-50 \mathrm{~cm})$. En todas las especies, la concentración radical disminuyó a medida que se incrementó la profundidad en ambos sitios. La presencia de raíces micorrizadas en las capas más profundas del suelo para las cuatro especies de Chenopodiaceae, muestran que estos hongos son tolerantes a vivir en los suelos salinos del centro de Argentina.

Palabras clave: Hongos micorrícico-arbusculares; ambientes salinos; perfil del suelo; distribución vertical.

\section{INTRODUCCIÓN}

En el Norte de la provincia de Córdoba y dentro de la vegetación correspondiente a las Salinas

\footnotetext{
1 Laboratorio de Micología, Instituto Multidisciplinario de Biología Vegetal (IMBIV) - CONICET- Universidad Nacional de Córdoba (UNC)- CC 495, 5000 Córdoba, República Argentina. Tel: 54-0351-433-4141, abecerra@ unc.edu.ar

2 Cátedra de Métodos Cuantitativos Aplicados, Facultad de Agronomía, Universidad de Buenos Aires (UBA). TelFax: 54-011-45248000, República Argentina.

3 Instituto Spegazzini. Facultad de Ciencias Naturales y Museo. Avenida 53, № 477. 1900 La Plata. Tel-Fax: 540221-4219845, República Argentina.
}

Grandes, las comunidades vegetales se ven reducidas a las zonas próximas al salar (Cabido \& Zak, 1999). Dentro de las especies halófitas adaptadas a la alta concentración de sales se encuentran especies de Chenopodiaceae (Cabido \& Zak, 1999). Estas plantas poseen mecanismos morfo-fisiológicos que permiten aprovechar de manera muy eficiente los escasos recursos nutricionales de los suelos salinos (Uchiyama, 1987; Khan et al., 2000). Una de las estrategias utilizadas por las halófitas para captar nutrientes, es la asociación con los microorganismos del suelo y en especial con los hongos micorrícico arbusculares (HMA), que permiten el crecimiento y desarrollo en condiciones estresantes (Hildebrandt et al., 2001; Plenchette \& Dupponois, 2005). Los HMA 
establecen una simbiosis con las raíces de las plantas lo que les permite explorar una mayor superficie del suelo, y de esta forma captar e incorporar los escasos nutrientes (Smith \& Read, 2008), protegiéndolas contra los patógenos radicales (Newsham et al., 1995), y ayudándolas a tolerar la sequía, la salinidad (Miller \& Jastrow, 1992) y los metales tóxicos contaminantes (Meharg \& Cairney, 2000).

Aunque las especies de Chenopodiaceae han sido consideradas como no micotróficas (Gerdemann, 1968; Hirrel et al., 1978; Peterson et al., 1985; Mohankumar \& Mahadevan, 1987), se ha observado que Salicornia sp., Suaeda maritima, Atriplex lampa y A. cordobensis están colonizadas por los HMA (Kim \& Weber, 1985; Rozema et al., 1986; Sengupta \& Chaudhari, 1990; Cofré et al., 2012; Soteras et al., 2013).

Los HMA usualmente están restringidos a los primeros 20 centímetros del suelo, donde se encuentra la mayor biomasa radical (Brundrett, 1991). En general, se ha observado una disminución de la colonización micorrícica (Rillig \& Field, 2003), en los propágulos infectivos (esporas, micelio extraradical y raíces micorrizadas) (An et al., 1990; Kabir et al., 1998) al aumentar la profundidad del suelo. Hasta ahora, solo un trabajo reporta para Atriplex lampa una colonización micorrícica similar a lo largo del perfil de suelo para las Salinas de Córdoba (Soteras et al., 2013).

En general, el sistema radical (raíces primarias y alimentadoras) de algunas Chenopodiaceae ocupan las capas superficiales del suelo (Barrow, 1997). Cuando el suelo lo permite, las raíces primarias llegan a más de $6 \mathrm{~m}$ de profundidad (Ogle \& St. John, 2003). El objetivo del presente trabajo fue estudiar la colonización micorrícica arbuscular y la concentración radical de cuatro especies de Chenopodiaceae (Allenrolfea patagonica (Moq.) Kuntze, Atriplex argentina Speg., Heterostachys ritteriana (Moq.) Moq. y Suaeda divaricata Moq.) en dos salinas del Centro de Argentina y en cinco profundidades del suelo $(0-50 \mathrm{~cm})$. Se hipotetiza que la colonización micorrícica y concentración radical están concentradas en los primeros centímetros del suelo.

\section{Material y Método}

Área de estudio y colección de muestras

El estudio se realizó en dos zonas del norte de la provincia de Córdoba, las Salinas de Ambargasta ( $\left.29^{\circ} 27^{\prime} 45,6^{\prime \prime} \mathrm{S}, 64^{\circ} 18^{\prime} 21,9^{\prime \prime} \mathrm{W}\right)$ perteneciente al Refugio de Vida Silvestre "Monte Las Barrancas" y

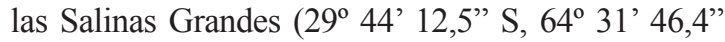
W) en la Reserva de Uso Múltiple "Salinas Grandes" (Departamentos de Tulumba, Ischilín y Cruz del Eje). El clima en ambos sitios es seco y cálido, con una precipitación media anual por debajo de los $500 \mathrm{~mm}$ y una temperatura media de $19,9{ }^{\circ} \mathrm{C}$. El área $(170$ msnm, con baja concentración de sales) está ocupada por vegetación xerofítica tales como Aspidosperma quebracho-blanco Schltdl., Prosopis flexuosa DC., Cercidium australe Johnst., Mimozyganthus carinatus (Grisseb.) Burkart, Ziziphus mistol Griseb., Prosopis torquata (Cav. ex Lag.) DC., y Stetsonia coryne (Salm-Dyck) Britton \& Rose.

El área de estudio, al borde del salar, muestra limitaciones para el desarrollo de cualquier tipo de cobertura vegetal, con la presencia de cuatro especies de Chenopodiaceae adaptadas a este ambiente: Allenrolfea patagonica, Atriplex argentina, Heterostachys ritteriana y Suaeda divaricata (Cabido \& Zak, 1999; Cabido et al., 2006). Las cuatro especies fueron muestreadas en un área de aproximadamente 50 x $50 \mathrm{~m}$ en cada uno de los dos sitios, durante finales del verano (primeros días de marzo 2007) aún las plantas se encontraban en su esplendor y con flores. Muestras de suelo y raíces de 5 plantas de cada especie fueron tomadas al azar con un sacabocado de metal $(3 \mathrm{~cm}$ de diámetro). Las 5 muestras de suelo y raíces se consideraron réplicas.

Las muestras de la rizosfera de cada planta se recolectaron entre los 0 y más de $40 \mathrm{~cm}$ de profundidad, a intervalos de $10 \mathrm{~cm}(0-10,10-20$, 20-30, 30-40, y 40-50 cm de profundidad) en cada sitio. Las muestras de suelo de cada profundidad (620 $\mathrm{cm}^{3}$ volumen de suelo) fueron colocadas en bolsas de plástico individuales y almacenadas a $4{ }^{\circ} \mathrm{C}$. En el laboratorio, las raíces finas de cada muestra fueron extraídas, lavadas con agua corriente y fijadas en FAA (formol: alcohol: ácido acético) para determinar posteriormente la colonización micorrícica.

A fin de caracterizar el suelo de cada sitio, a las 5 muestras tomadas por nivel de profundidad se les midieron los siguientes parámetros edáficos: conductividad eléctrica $(\mathrm{mmhos} / \mathrm{cm})$, fósforo extraíble determinado por el método de Bray and Kurtz I (Jackson, 1964), pH, contenido de materia orgánica siguiendo la metodología de Nelson \& 


\section{A. Becerra et al. - Hongos micorrícico-arbusculares en Chenopodiaceae}

Sommers (1982), relación carbono:nitrógeno y textura del suelo. El nitrógeno total fue determinado usando el método de micro-Kjeldhal (Bremner \& Mulvaney, 1982). Las características edáficas se detallan en la Tabla 1.

\section{Colonización micorrícica arbusculary concentración radical}

Raíces frescas previamente lavadas con agua, se aclararon con $\mathrm{KOH} 10 \%\left(15 \mathrm{~min}\right.$ a $\left.90{ }^{\circ} \mathrm{C}\right)$, luego se clarificaron con $\mathrm{H}_{2} \mathrm{O}_{2}$ al 30\% (10 min, temperatura ambiente), se acidificaron con $\mathrm{HCl} 1 \%$ (1 min, temperatura ambiente) y tiñeron durante 5 min en azul de tripán al 0.05\% (Phillips \& Hayman, 1970). El porcentaje del largo de la raíz colonizada fue determinado con el método de intersección de la cuadrícula (Giovannetti \& Mosse, 1980) bajo lupa esteroscópica Leica M 420. Para cada una de las especies vegetales y por profundidad se analizaron 100 segmentos radicales de $1 \mathrm{~cm}$ de largo. La colonización micorrícica arbuscular (CMA) fue clasificada en 5 categorías: muy alta $(>80 \%)$, alta (60$79 \%$ ), media (40-59\%), baja (20-39\%) y muy baja (1-19\%) (Zangaro et al., 2002).

Para cada profundidad muestreada, se cuantificó la concentración radical (número de segmentos radicales de $1 \mathrm{~cm}$ de longitud en $100 \mathrm{~cm}^{3}$ de volumen de suelo).

\section{Análisis estadísticos}

Se aplicó un análisis de la varianza (ANAVA) de medidas repetidas sobre los datos a fin de evaluar el efecto de los niveles de profundidad del suelo $(0-10,10-20,20-30,30-40$, y $40-50 \mathrm{~cm}$ - factor intra-sujeto), los sitios y las especies hospedantes (factores entre-sujetos) sobre los parámetros del suelo, la CMA y la concentración radical. Se aplicó un test post-hoc de comparaciones múltiples de Bonferroni o Tukey HSD (nivel de significancia de $0,05)$ para determinar la diferencia entre los niveles del factor intra-sujeto. Se comprobaron los supuestos de normalidad y homogeneidad de los residuales (Shapiro-Wilks y Levene respectivamente). Se aplicó una transformación logarítmica a la variable concentración radical. Se evaluó la relación entre la concentración de raíces y la CMA para cada profundidad y sitio a través de análisis de correlación de Pearson. Todos los datos se analizaron utilizando el programa estadístico STATISTICA (http://www. statsoft.com/).

\section{Resultados}

\section{Características del suelo}

Los suelos de las Salinas de Ambargasta (SA) y las Salinas Grandes (SG) son Salortides típicos (Jarsún et al., 2003). Ambos suelos presentaron una textura franco arcillo arenosa, alto $\mathrm{pH}$, elevada conductividad eléctrica y bajo contenido de materia orgánica. Los suelos de la SA son fuertemente alcalinos con un nivel de $\mathrm{P}$ muy bajo $(<5 \mathrm{ppm})$; mientras que los de la SG son moderadamente alcalinos y un nivel de $\mathrm{P}$ bajo (5-12 ppm). En ambos sitios se observó una disminución de la materia orgánica, sólo significativa en las SA, y un incremento de la conductividad eléctrica con la profundidad del suelo (no significativo). La relación $\mathrm{C} / \mathrm{N}$ en las $\mathrm{SA}$ fue significativamente menor de $0-10 \mathrm{~cm}$ que de $40-50 \mathrm{~cm}$. En las $\mathrm{SG}$ la relación $\mathrm{C} / \mathrm{N}$ no fue significativa (Tabla 1 ).

\section{Colonización micorrícica}

Las raíces de Atriplex argentina, Allenrolfea patagonica, Heterostachys ritteriana y Suaeda divaricata presentaron colonización micorrícica arbuscular (puntos de entrada, circunvoluciones, hifas intra- e inter-celulares y vesículas intra- e inter-celulares) en ambos sitios y en todas las profundidades (Fig. 1). No se observaron arbúsculos en ninguno de los hospedantes estudiados.

La CMA en ambos sitios varió desde muy bajo a medio; en las Salinas Grandes (SG) de 2 a $50 \%$ y en las Salinas de Ambargasta (SA) de 0 a $45 \%$. La colonización varió a lo largo del perfil del suelo (0-10 cm: $12-45 \% ; 10-20 \mathrm{~cm}: 5-50 \% ; 20-30 \mathrm{~cm}$ : 0-45\%; 30-40 cm: 4-45\%; 40-50 cm: 0-30\%). La CMA en $A$. patagonica y $S$. divaricata fue muy baja a baja (5-31\% y $2-37 \%$, respectivamente) y en $H$. ritteriana y $A$. argentina fue muy baja a media (0-45\% y $4-50 \%$, respectivamente). La CMA difirió entre las especies de plantas a través de las profundidades del suelo (Tabla 2). Se observaron interacciones significativas entre las profundidades del suelo y las especies de plantas y entre las profundidades del suelo y los sitios (Tabla 2). Atriplex argentina presentó el mayor porcentaje de CMA en casi todas las profundidades para ambos sitios y fue significativamente diferente a las otras especies (Fig. 2).

La concentración radical disminuyó a lo largo del perfil del suelo (valores expresados en $\mathrm{cm}$ 
Tabla 1. Propiedades del suelo de las Salinas de Ambargasta (SA) y las Salinas Grandes (SG), a cinco profundidades de suelo $(0-10,10-20,20-30,30-40$, y $40-50 \mathrm{~cm})$. Los valores son la media de 5 muestras \pm error estándar. Los valores dentro de cada fila con letras distintas son significativamente diferentes según el test de Tukey HSD para un $P<0,05$.

\begin{tabular}{|c|c|c|c|c|c|c|}
\hline & & $0-10 \mathrm{~cm}$ & $10-20 \mathrm{~cm}$ & $20-30 \mathrm{~cm}$ & $30-40 \mathrm{~cm}$ & $40-50 \mathrm{~cm}$ \\
\hline \multirow{2}{*}{$\mathrm{MO}^{1}(\%)$} & SA & $1,29 \pm 0,40 a$ & $0,81 \pm 0,18 a b$ & $0,82 \pm 0,17 a b$ & $0,71 \pm 0,21 b$ & $0,65 \pm 0,23 b$ \\
\hline & SG & $2,30 \pm 1,93 a$ & $1,41 \pm 1,06 a$ & $1,04 \pm 0,61 \mathrm{a}$ & $1,05 \pm 0,71 \mathrm{a}$ & $0,86 \pm 0,46 a$ \\
\hline \multirow{2}{*}{$\mathrm{C} / \mathrm{N}^{2}$} & SA & $8,24 \pm 1,72 \mathrm{a}$ & $7,90 \pm 0,62 a$ & $10,26 \pm 2,57 a b$ & $10,47 \pm 2,88 a b$ & $12,93 \pm 2,59 b$ \\
\hline & SG & $9,79 \pm 2,35 a$ & $9,23 \pm 2,48 a$ & $10,91 \pm 3,63 a$ & $11,95 \pm 2,04 \mathrm{a}$ & $12,52 \pm 0,65 a$ \\
\hline \multirow{2}{*}{ Fósforo (ppm) } & SA & $11,61 \pm 6,74 a$ & $10,89 \pm 7,93 a$ & $8,53 \pm 5,55 a$ & $8,76 \pm 5,65 a$ & $9,17 \pm 4,81 \mathrm{a}$ \\
\hline & SG & $7,04 \pm 3,39 a$ & $6,48 \pm 2,68 a$ & $6,60 \pm 3,40 a$ & $7,17 \pm 4,15 a$ & $9,93 \pm 4,40 a$ \\
\hline \multirow{2}{*}{ pH 1: 2,5} & SA & $8,17 \pm 0,57 \mathrm{a}$ & $8,11 \pm 0,50 a$ & $8,07 \pm 0,42 a$ & $8,02 \pm 0,37 a$ & $8,17 \pm 0,50 a$ \\
\hline & SG & $7,76 \pm 0,16 \mathrm{a}$ & $7,89 \pm 0,19 a$ & $7,84 \pm 0,10 a$ & $7,89 \pm 0,19 a$ & $7,82 \pm 0,09 a$ \\
\hline \multirow{2}{*}{$\mathrm{CE}^{3}\left(\mathrm{dS} \cdot \mathrm{m}^{-1}\right)$} & SA & $11,45 \pm 5,79 a$ & $13,53 \pm 4,53 a$ & $17,19 \pm 5,12 a$ & $17,58 \pm 3,90 a$ & $18,85 \pm 4,86 a$ \\
\hline & SG & $9,35 \pm 5,56 a$ & $12,48 \pm 6,05 a$ & $14,36 \pm 4,04 a$ & $11,98 \pm 5,48 a$ & $14,91 \pm 3,42 \mathrm{a}$ \\
\hline
\end{tabular}

${ }^{1} \mathrm{MO}$ : Materia orgánica

${ }^{2} \mathrm{C} / \mathrm{N}$ : Relación carbono: nitrógeno

${ }^{3} \mathrm{CE}$ : Conductividad eléctrica

cada $100 \mathrm{~cm}^{3}$ ): $0-10 \mathrm{~cm}: 7-139 ; 10-20 \mathrm{~cm}: 5-119$; 20-30 cm: 4-38; 30-40 cm: 3-23; 40-50 cm: 1-13. El ANAVA de medidas repetidas mostró que esta variable varió significativamente a través del perfil del suelo (Tabla 2). Se observaron interacciones significativas entre especies hospedantes y sitios, y una interacción tripartita entre profundidad del suelo x plantas x sitios (Tabla 2), indicando que en todas las especies la concentración radical disminuyó a medida que aumentó la profundidad en ambos sitios.

La correlación de Pearson entre la CMA y la concentración radical fue positiva y significativa en dos profundidades para la SA $(10-20 \mathrm{~cm} \mathrm{r}=0,62 ; p$ $<0,01 ; 40-50 \mathrm{~cm} \mathrm{r}=0,45 ; p<0,05)$. Para el resto de las profundidades las correlaciones fueron positivas no significativas. En las SG las correlaciones fueron negativas no significativas, excepto en la profundidad $0-10 \mathrm{~cm}$ que resultó ser positiva no significativa.

\section{Discusión}

El presente trabajo muestra la distribución vertical de la colonización micorrícica y la concentración radical en cada una de las 4 especies de chenopodiaceae analizadas y categorizadas en la literatura como no micotróficas (Gerdemann, 1968; Peterson et al., 1985). La presencia de estructuras micorrícicas arbusculares en estas plantas coincide con lo citado en la literatura (Allen, 1983; Fontenla et al., 2001; Plenchette \& Duponnois, 2005), especialmente bajo condiciones de estrés tales como la sequía y la salinidad (Hirrel, 1981; Rozema et al., 1986; Sengupta \& Chaudhuri, 1990; Cofré et al., 2012; Soteras et al., 2013).

Las cuatro especies estudiadas mostraron en sus raíces las estructuras típicas de los Glomeromycota, aunque no se observaron arbúsculos. Los arbúsculos han sido considerados como el sitio de intercambio de carbono y fósforo entre las células radicales y la 

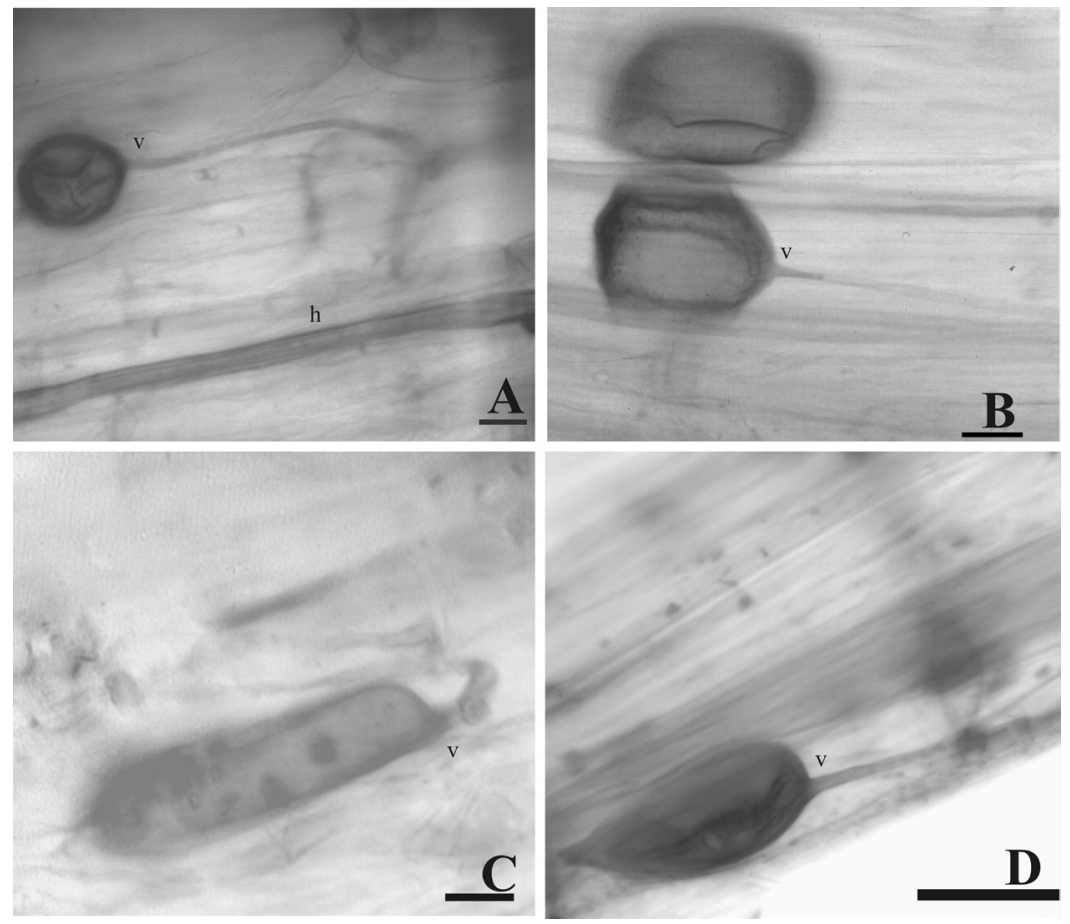

Fig. 1. Colonización micorrícica arbuscular en Chenopodiaceae. A: Vesículas intracelulares (v) e hifa intercelular (h) en Allenrolfea patagonica. B: Vesículas intracelulares (v) en Atriplex argentina. C: Vesículas intercelulares (v) en Heterostachys ritteriana. D: Vesículas intracelulares (v) en Suaeda divaricata. Escala

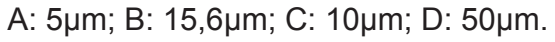

hifas de los Glomeromycota (Brundrett, 2002). Estos resultados pueden sugerir que las circunvoluciones y/o las hifas intra-radicales, presentes en las raíces de las plantas estudiadas, pueden estar jugando un rol importante en el intercambio de carbono/ fósforo en las Chenopodiaceae (Plenchette \& Duponnois, 2005; Smith \& Smith, 2011; Zhang et al., 2012; Soteras et al., 2013). Para Manjarrez et al. (2010) los arbúsculos no son un requerimiento en la transferencia de fósforo a las plantas; por lo que se necesitan más estudios para determinar los efectos benéficos de los hongos micorrícicos a las Chenopodiaceae.

Los valores de colonización micorrícica de las plantas muestreadas fueron similares a los reportados para otras plantas de suelos salinos (Brown \& Bledsoe, 1996; Udaiyan et al., 1996; Hilderbrandt et al., 2001; Landwerh et al., 2002; Cofré et al., 2012; Soteras et al., 2013). De las cuatro especies estudiadas, A. argentina presentó el mayor \% de CMA en ambos sitios. Esto coincide con lo encontrado por Soteras et al. (2013) para A. lampa en los mismos sitios bajo estudio (para SG: 49,02 \%, para SA: 30,31\%) y por Cofré et al. (2012) para A. cordobensis (SG: 59,95\%).

La CMA y la concentración radical variaron a lo largo del perfil del suelo. Zajicek \& Hetrick (1986) observaron que el grado de colonización varía con las especies de plantas y con la profundidad del suelo sugiriendo que el crecimiento de las raíces en profundidad puede ser, para las plantas, un mecanismo de supervivencia por la competencia de agua y nutrientes, ya que la fertilidad del suelo generalmente disminuye con el incremento en profundidad (Hayes \& Seasted, 1989; Lai et al., 2014; Sala et al., 1992). Esto coincide con lo encontrado en este trabajo ya que se observaron diferencias en los parámetros edáficos a medida que se incrementó la profundidad (una reducción de $\mathrm{MO}, \mathrm{C}$ y $\mathrm{N}$ en SA y una reducción de $\mathrm{K}$ y un incremento de $\mathrm{Na}$ en $\mathrm{SG}$ ) lo que probablemente afecte la CMA, tal como ha sido observado en otras 

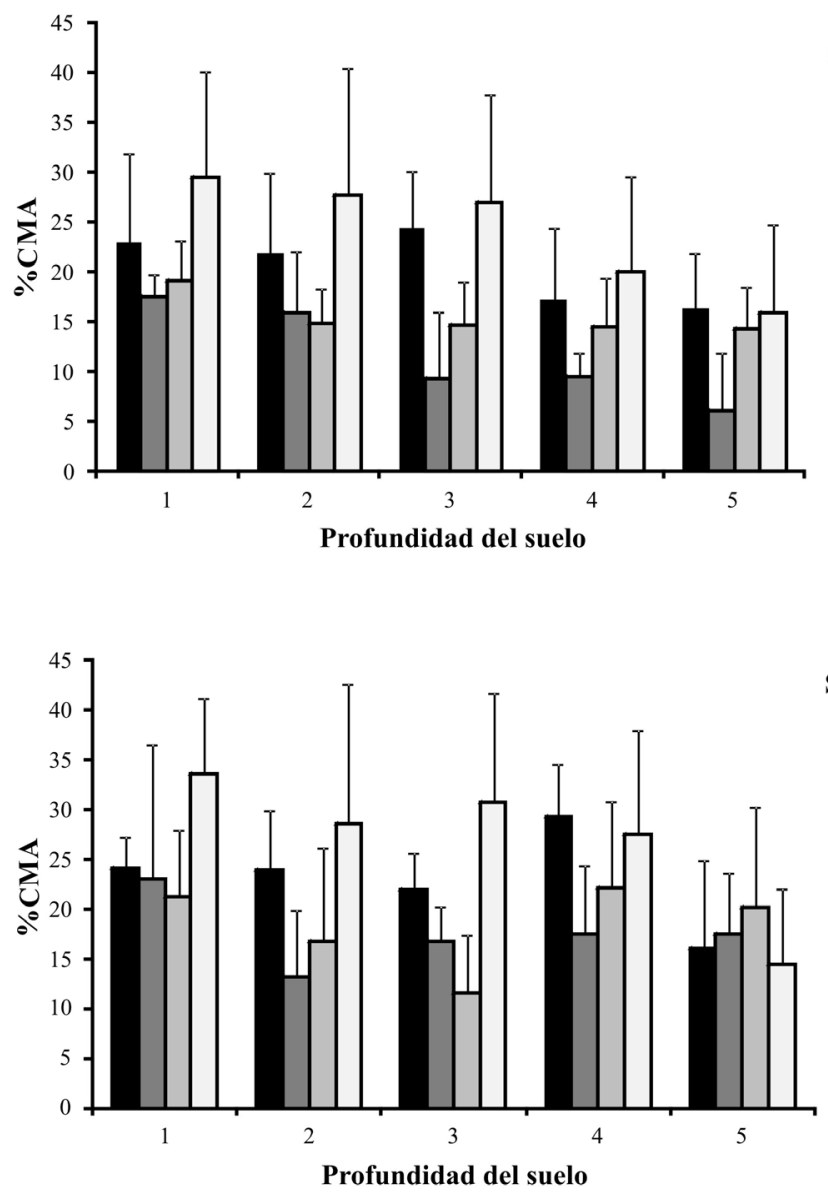

Fig. 2. Colonización micorrícica arbuscular (\% CMA) en los cuatro hospedantes estudiados para las diferentes profundidades del suelo en ambos sitios (Salinas de Ambargasta (SA), Salinas Grandes (SG)). Los valores corresponden al promedio de 5 muestras \pm error estándar. Hospedantes: Allenrolfea patagonica $(\square)$, Atriplex argentina ( ), Heterostachys ritteriana $(\square)$ y Suaeda divaricata $(\square)$. Profundidades del suelo $1(0-10 \mathrm{~cm}), 2(10-20 \mathrm{~cm}), 3(20-30 \mathrm{~cm}), 4(30-40 \mathrm{~cm}), 5(40-50 \mathrm{~cm})$.

especies de Chenopodiaceae (Aguilera et al., 1998).

Se observaron correlaciones positivas entre la CMA y la concentración radical para la Salinas de Ambargasta en todas las profundidades, resultando significativa a los $10-20 \mathrm{~cm}$ y $40-50 \mathrm{~cm}$ de profundidad del suelo. Para las Salinas Grandes las correlaciones fueron negativas no significativas, excepto para la profundidad $0-10 \mathrm{~cm}$ que resultó positiva. La concentración radical disminuyó a lo largo del perfil del suelo, y aunque las raíces de estas plantas pueden extenderse hasta los $6 \mathrm{~m}$ de profundidad (Ogle \& St. John 2003), se observó CMA en las raíces de los hospedantes a lo largo de todo el perfil del suelo. Los propágulos infectivos se encuentran concentrados generalmente en los horizontes superficiales del suelo (Oehl et al., 2005) aunque también pueden encontrarse a mayores profundidades (Muthukumar et al., 2003; Wang et al., 2004; Becerra et al., 2014) lo que explicaría la colonización en capas más profundas del suelo.

El presente estudio contribuye con el conocimiento de las micorrizas arbusculares de las Chenopodiaceae creciendo en ecosistemas salinos naturales. Los resultados muestran una colonización micorrícica y una concentración radical alta en la superficie del suelo disminuyendo a medida que se incrementa la profundidad. Estos resultados apoyan nuestra hipótesis. Además, en estos mismos 
Tabla 2. Valores F del ANAVA de medidas repetidas para la Colonización Micorrícica Arbuscular (\%CMA) y Concentración de Raíces (CR) en las plantas hospedantes estudiadas (PH), sitios (S) (Salinas de Ambargasta y Salinas Grandes) y profundidades del suelo (PS).

${ }^{*} P<0,05 .{ }^{* * *} P<0,001$.

\begin{tabular}{|lcc|}
\hline & $\%$ CMA & CR \\
\hline Efecto intra-sujeto & & $109,6824^{* * *}$ \\
Profundidad del suelo (PS) & $11,9322^{* * *}$ & 1,247 \\
PS x PH & $3,0376^{* * *}$ & 0,418 \\
PS x S & $3,1319^{*}$ & $2,0800^{*}$ \\
PS x PH x S & 1,192 & \\
Efecto entre-sujetos & & 0,979 \\
Plantas hospedantes (PH) & $6,9942^{* * *}$ & 0,223 \\
Sitios (S) & 3,736 & $3,2476^{*}$ \\
PH x S & 0,178 & \\
\hline
\end{tabular}

sitios se ha observado una alta diversidad de HMA pertenecientes a diferentes géneros y familias de Glomeromycota (Becerra et al., 2014; Soteras et al., 2012), por lo que se sugiere que estos hongos junto a sus hospedantes estarían adaptados a condiciones medioambientales extremas de $\mathrm{pH}$ y salinidad (Wang et al., 2004).

\section{Agradecimientos}

Este trabajo estuvo financiado por la Secretaría de Ciencia y Técnica -Universidad Nacional de Córdoba- y la Agencia de Promoción Científica y Tecnológica (PICT 438-2006) y CICPBA para los análisis del suelo. A.B. es investigadora del Consejo Nacional de Investigaciones Científicas y Tecnológicas (CONICET). M.C. es investigadora de la Comisión de Investigaciones Científicas de la provincia de Buenos Aires (CICPBA). F.S. y N.C. son becarias postdoctorales de CONICET.

\section{Bibliografía}

AGUILERA, L. E., J. R. GUTIERREZ \& R. J. MORENO. 1998. Vesiculo arbuscular mycorrhizae associated with saltbushes Atriplex spp. (Chenopodiaceae) in the Chilean Arid Zone. Rev. Chil. Hist. Nat. 71: 291-302.
ALLEN, M. F. 1983. Formation of vesicular arbuscular mycorrhizae in Atriplex gardneri (Chenopodiaceae): seasonal response in a cold desert. Mycologia 75: 773-776.

AN, Z. Q., J. H. GROVE, J. W. HENDRIX, D. E. HERSHMAN \& G. T. HENSON. 1990. Vertical distribution of endogonaceous mycorrhizal fungi associated with soybean, as affected by soil fumigation. Soil Biol. Biochem. 22: 715-719.

BARROW, J. R. 1997. Natural asexual reproduction in fourwing saltbrush Atriplex canescens (Pursh) Nutt. J. Arid Environ. 36: 267-270.

BECERRA, A., J. BARTOLONI, N. COFRÉ, F. SOTERAS \& M. CABELLO. 2014. Arbuscular mycorrhizal fungi in saline soils: vertical distribution at different soil depths. Braz. J. Microbiol. 45: 585594.

BREMNER, J. M. \& C. S. MULVANEY. 1982. Nitrogen total. In: PAGE, A. L. (ed.), Methods of soil analysis Part II, pp. 595-562. American Society of Agronomy: Soil Science Society of America, Madison, Wisconsin.

BROWN, A. M. \& C. BLEDSOE. 1996. Spatial and temporal dynamics of mycorrhizas in Jaumea carnosa, a tidal saltmarsh halophyte. J. Ecol. 84: 703-715.

BRUNDRETT, M. 1991. Mycorrhizas in natural ecosystems. In: MACFAYDEN, A., M. BEGON \& A. H. FITTER (eds.), Advances in Ecological Research, vol. 21, pp. 171-262. Academic Press, London.

BRUNDRETT, M.C. 2002. Coevolution of roots and 
mycorrhizas of land plants. New Phytol. 154: 275304.

CABIDO, M. \& M. ZAK. 1999. Vegetación del Norte de Córdoba. Córdoba: Secretaría de Agricultura, Ganadería y Recursos Renovables de Córdoba. 56 pp. ISBN: 987-43-0942-3.

CABIDO, M., C. ROSACHER, J. M. ROQUÉ GARZÓN, D. CABIDO, J. GORGAS \& J. PIANA. 2006. Reserva de Usos Múltiples Salinas Grandes. En: CABIDO, M., C. ROSACHER, J. M. ROQUÉ GARZÓN \& D. CABIDO (eds.), Áreas Naturales Protegidas de la Provincia de Córdoba, pp. 81-96. Agencia Córdoba Ambiente, Gobierno de Córdoba, La Voz del Interior.

COFRÉ, N., A. BECERRA, E. NOUHRA \& F. SOTERAS. 2012. Arbuscular mycorrhizae and dark-septate endophytes on Atriplex cordobensis in saline sites from Argentina. J. Agric. Technol. 87(7): 2201-2214.

FONTENLA, S., E. CHAIA, C. BUSTOS \& A. PELLIZA. 2001. Microorganismos simbióticos en Atriplex. XXVIII Jornadas Argentinas de Botánica. Santa Rosa, La Pampa, Argentina. 21 al 25 de Octubre. Bol. Soc. Arg. Bot. 36: 114.

GERDEMANN, J. W. 1968. Vesicular arbuscular mycorrhiza and plant growth. Ann. Rev. Phytopatol. 6: 397-418.

GIOVANNETTI, M. \& B. MOSSE. 1980. An evaluation of techniques for measuring vesicular-arbuscular mycorrhizal infection in roots. New Phytol. 84: 489500 .

HAYES, D. C. \& T. R. SEASTED. 1989. Nitrogen dynamics of soil water in burned and unburned tallgrass prairie. Soil. Biol. Biochem. 21: 1003-1007.

HILDEBRANDT, U., K. JANETTA, O. FOUAD, B. RENNE, K. NAWRATH \& H. BOTHE. 2001. Arbuscular mycorrhizal colonization of halophytes in Central European salt marshes. Mycorrhiza 10: 175-183.

HIRREL, M.C. 1981. The effect of sodium and chloride salts on the germination of Gigaspora margarita. Mycologia 43: 610-617.

HIRREL, M. C., H. MEHRAVARAN \& J. W. GERDEMANN. 1978. Vesicular-arbuscular mycorrhizae in the Chenopodiaceae and Cruciferae: do they occur? Can. J. Bot. 56: 2813-2817.

JACKSON, M. L. 1964. Análisis químico de suelos. 2nd Edn. Omega, Barcelona.

JARSÚN, B., J. A. GORGAS, E. ZAMORA, E. BOSNERO, E. LOVERO, A. RAVELO \& J. L. TASSILE. 2003. Recursos Naturales de La Provincia de Córdoba: Los Suelos. Nivel de Reconocimiento 1:500.000. Argentina. Agencia Córdoba D.A.C. y T.S.E.M Dirección de Ambiente e INTA (INSTITUTO NACIONAL DE TECNOLOGÍA AGROPECUARIA-MANFREDI).
KABIR, Z., I. P. O'HALLORAN, P. WIDDEN \& E. HAMEL. 1998. Vertical distribution of arbuscular mycorrhizal fungi under corn (Zea mays L.) in notill and conventional tillage systems. Mycorrhiza 8: 53-55.

KHAN, M. A., I. A. UNGAR, \& A. M. SHOWALTER. 2000. Effects of salinity on growth, water relations and ion accumulation of the subtropical perennial halophyte, Atriplex griffithii var. stocksii. Ann. Bot. 85: 225-232.

KIM, C-K. \& D. J. WEBER. 1985. Distribution of VA mycorrhiza on halophytes on inland salt playas. Plant Soil 83: 207-214.

LAI, Z., Y. ZHANG, B. WU, T. ZHA, S. QIN, X. JIA, J. LIU \& W. FENG 2014. Fine root distribution and belowground interactions in an alley silvopasture system in northern China. Turk. J. Agric. For. 38:644-651.

LANDWEHR, M., U. HIDELBRANDT, P. WILDE, K. NAWRATH, T. TOTH, B. BIRO \& H. BOTHE. 2002. The arbuscular mycorrhizal fungus Glomus geosporum in European saline, sodic and gypsum soils. Mycorrhiza 12: 199-211.

MANJARREZ, M., H.M. CHRISTOPHERSEN, S. E. SMITH \& F.A. SMITH. 2010. Cortical colonization is not an absolute requirement for phosphorus transfer to plants in arbuscular mycorrhizas formed by Scutellospora calospora in tomato mutant: evidence from physiology and gene expression. Funct. Plant Biol. 37:1132-1142.

MEHARG, A. A. \& J. W. G. CAIRNEY. 2000. Coevolution of mycorrhizal symbionts and their hosts to metal-contaminated environments. Adv. Ecol. Res. 30:70-102.

MOHANKUMAR, V. \& A. MAHADEVAN. 1987. Vesicular-arbuscular mycorrhizal association in plants of Kalakad reserve forest, India. Angew. Bot. 61: 255-274.

MILLER, R. M. \& J. D. JASTROW. 1992. The application of VA mycorrhizae to ecosystem restoration and reclamation. In: ALIEN M.P. (ed), Mycorrhizal Functioning, pp. 438-467. Chapman \& Hall, New York.

MUTHUKUMAR T., L. SHA, X. YANG, M. CAO, J. TANG, Z. ZHENG. 2003. Distribution of roots and arbuscular mycorrhizal associations in tropical forest types of Xishuangbanna, southwest China. App. Soil Ecol. 22: 241-253.

NELSON, D. W. \& L. E SOMMERS. 1982. Total carbon, organic carbon, and organic matter. In: PAGE, A. L., R. H. MILLER \& D. R. KEENEY (eds.), Methods of soil analysis, Part 2, pp. 639-577. Amer. Soc. Agron., Inc., Madison.

NEWSHMAN, K. K., A. H. FITTER \& A. R. WATKINSON. 1995. Multi-functionality and 


\section{A. Becerra et al. - Hongos micorrícico-arbusculares en Chenopodiaceae}

biodiversity in arbuscular mycorrhiza. Trends Ecol. Evol. 10: 407-411.

OEHL, F, E. SIEVERDING, K. INEICHEN, E. A. RIS, T. BOLLER \& A. WIEMKEN. 2005. Community structure of arbuscular mycorrhizal fungi at different soil depths in extensively and intensively managed agroecosystems. New Phytol. 165: 273-283.

OGLE, D. G. \& L. St. JOHN. 2003. Plant Guide: Fourwing saltbush Atriplex canescens (Pursh) Nutt. In: Plants Database. U.S. Department of Agriculture, Natural Resources Conservation Service (Producer). http://plants.usda.gov/plantguide/pdf/pg_atca2.pdf

PETERSON, R. L., A. E. ASHFORD \& W. G. ALLAWAY. 1985. Vesicular-arbuscular mycorrhizal association of vascular plants on Heron Island, a great barrier reef coral ray. Aust. J. Bot. 33: 669-676.

PHILLIPS, J. M. \& D. S. HAYMAN. 1970. Improved procedures for clearing roots and staining parasitic and vesicular-arbuscular mycorrhizal fungi for rapid assessment of infection. Trans. Br. Mycol. Soc. 55: $158-161$.

PLENCHETTE, C. \& R. DUPONNOIS. 2005. Growth response of the saltbush Atriplex nummularia L. to inoculation with the arbuscular mycorrhizal fungus Glomus intraradices. J. Arid Environ. 61: 535-540.

RILliG, M. C. \& C. B. FIELD, 2003. Arbuscular mycorrhizae respond to plants exposed to elevated atmospheric $\mathrm{CO}_{2}$ as a function of soil depth. Plant Soil 254: 383-391.

ROZEMA, J., W. ARP, J. VAN DIGGELEN, M. VAN ESBROEK, R. BROEKMANN \& H. PUNTE. 1986. Occurrence and ecological significance of vesicular arbuscular mycorrhiza in the salt marsh environment. Acta Bot. Neerland. 35: 457-467.

SALA, O. E., W. K. LAUENORTH \& W. J. PARTON. 1992. Long-term soil water dynamics in the shortgrass steppe. Ecology 73: 1175-1181.

SENGUPTA, A. \& S. CHAUDHURI. 1990. Vesicular arbuscular mycorrhiza (VAM) in pioneer salt marsh plants of the Ganges River delta in West Bengal (India). Plant Soil 122: 111-113.

SOTERAS, F., A. BECERRA, N. COFRÉ, J. BARTOLONI \& M. CABELLO. 2012. Arbuscular mycorrhizal fungal species in saline environments of Central Argentina: seasonal variation and distribution of spores at different soil depth. Sydowia 64: 301-311.
SOTERAS, F., N. COFRÉ, J. BARTOLONI, M. CABELLO \& A. BECERRA. 2013. Colonización radical de Atriplex lampa en dos ambientes salinos de Córdoba, Argentina. Bol. Soc. Arg. Bot. 48: 211-219.

SMITH, S. E. \& D. J. READ. 2008. Mycorrhizal Symbiosis. 3rd Edn. Academic Press, Elsevier, Amsterdam.

SMITH, S. E. \& F. A. SMITH. 2011. Roles of arbuscular mycorrhizas in plant nutrition and growth: new paradigms from cellular to ecosystems scales. Annu. Rev. Plant Biol. 62: 227-250.

UCHIYAMA, Y. 1987. Salt tolerance of Atriplex nummularia. Tech. Bull. Trop. Agric. Res. Center Jap. 22: 1-69.

UDAIYAN, K., A. KARTHIKEYAN \& T. MUTHUKUMAR. 1996. Influence of edaphic and climatic factors on dynamics of root colonization and spore density of vesicular-arbuscular mycorrhizal fungi in Acacia farnesiana Willd. and A. planifrons W. et A. Mycorrhiza 11: 65-71.

WANG, F. Y., R. J. LIU, X. G. LIN \& J. M. ZHOU. 2004. Arbuscular mycorrhizal status of wild plants in saline-alkaline soils of the Yellow River Delta. Mycorrhiza 14: 133-137.

ZAJICEK, J. M. \& B. A. D. HETRICK. 1986. The influence of soil depth on mycorrhizal colonization of forbs in the tallgrass prairie. Mycologia 78: 316320 .

ZHANG, T., N. SHI, D. BAI, Y. CHEN \& G. FENG. 2012. Arbuscular mycorrhizal fungi promote the growth of Ceratocarpus arenarius (Chenopodiaceae) with no enhacement of phosphorus nutrition. Plos One 7: e41151.

ZANGARO, W., S. M. A. NISIZAKI, J. C. B. DOMINGOS, E. M. NAKANO. 2002. Arbuscular mycorrhizal in native woody species of Tibagi River Basin, Paraná. Cerne 8: 77-87.

Recibido el 4 de febrero de 2015, aceptado el 30 de noviembre de 2015. 
